\title{
Health-related quality of life in patients with chronic hepatitis $B$ during antiviral treatment and off-treatment
}

This article was published in the following Dove Press journal:

Patient Preference and Adherence

12 January 2017

Number of times this article has been viewed

\section{Xiulan Xue ${ }^{1, *}$ \\ Shaohang Cai ${ }^{1-3, *}$ \\ Hongjie Ou' \\ Caixia Zheng' \\ Xiaolu Wu'}

'Department of Infectious Diseases, First Affiliated Hospital of Xiamen University, Xiamen, Fujian Province, ${ }^{2}$ Department of Pathology, Sun Yatsen University Cancer Center, ${ }^{3}$ State Key Laboratory of Oncology in South China, Collaborative Innovation Center for Cancer Medicine, Guangzhou, Guangdong Province, People's Republic of China

*These authors contributed equally to this work
Correspondence: Shaohang Cai Department of Pathology, Sun Yat-sen University Cancer Center, State Key Laboratory of Oncology in South China, Collaborative Innovation Center for Cancer Medicine, 65I Dongfeng East Rd, Yuexiu District, 510515 Guangdong, People's Republic of China

$\mathrm{Tel} / \mathrm{fax}+865926248021$

Email shaohangcai@foxmail.com
Introduction: Health-related quality of life (HRQoL) has emerged as an important consideration in the care of patients with chronic hepatitis B (CHB). However, whether benefits from the improved HRQoL that occurs after antiviral treatment or drug discontinuation outweigh the risks of viral relapse is an unanswered question. The aim of this study was to evaluate the HRQoL among patients with CHB during antiviral treatment and withdrawal of treatment.

Patients and methods: There were 102 patients who met the enrollment criteria with 54 patients in the treatment group and 48 patients in the discontinuation group. Sociodemographic information was collected. The 36-Item Short-Form Health Survey (SF-36), European Quality of Life-5 Dimensions, and Beck Depression Inventory (BDI) were adopted to evaluate life quality and mental health.

Results: In the treatment group, SF-36 showed that the physical functions were significantly increased. In the discontination group, the psychological functions showed improvement. A multivariate regression analysis indicated that baseline SF-36 score was a predictor for improvement in HRQoL (odds ratio $=1.17, P=0.003$ ) and baseline BDI score was a factor for remission of depression (odds ratio $=0.75, P=0.005$ ) after medical intervention. When the cutoff value of SF-36 score was set at 79.5, the sensitivity and specificity to predict improvement in HRQoL were $82.8 \%$ and $74.0 \%$, respectively. When the cutoff value of BDI was found as 8.5 , the sensitivity and specificity to predict alleviation of depression were $58.6 \%$, and $76.0 \%$, respectively.

Conclusion: Antiviral treatment benefits the physical health of the patients with $\mathrm{CHB}$, while conferring no obvious improvement in their psychological condition. Improved psychological interventions for patients with CHB, especially for those with lower baseline SF-36 scores and higher BDI scores, may improve their quality of life.

Keywords: chronic hepatitis B, health-related quality of life, antiviral treatment, mental disorder, depression

\section{Introduction}

Approximately 2 billion people worldwide have been infected with hepatitis $B$ virus (HBV), and $\sim 5 \%$ of them have chronic infections. ${ }^{1}$ Patients with chronic hepatitis B $(\mathrm{CHB})$ are at increased risk of developing end-stage liver diseases such as cirrhosis, liver failure, and hepatocellular carcinoma (HCC). ${ }^{2,3}$ Antiviral treatment can suppress HBV replication and improve the survival rate. Nucleos(t)ide analogs (NUCs) are safe and effective antiviral drugs, and they are recommended for first-line treatment of CHB by most guidelines. ${ }^{2,4}$ Although NUCs are potent inhibitors of HBV replication, they do not eliminate $\mathrm{HBV}$. Before starting long-term NUC treatment in patients with $\mathrm{CHB}$, issues of compliance, economic burden, and quality of life 
(QoL) should be taken into consideration, especially in low-income populations. ${ }^{5}$

Despite remarkable progress in the use of NUCs for the treatment of CHB, the effects of therapy with these drugs on the QoL and mental health $(\mathrm{MH})$ of patients have received limited attention. As modern medical practice has evolved from traditional biomedical models to modern biopsychosocial medical paradigms, health-related quality of life (HRQoL) has emerged as an important consideration in the care of patients with CHB. ${ }^{6}$ Chronic diseases, and especially $\mathrm{CHB}$, impose a heavy psychosocial burden, including worse HRQoL, depression, and anxiety. A study reported that HBV carriers have significantly lower social function than healthy controls. ${ }^{7}$ Furthermore, it has been reported that HRQoL of patients, as assessed by European Quality of Life-5 Dimensions (EQ-5D) and 36-Item Short-Form Health Survey (SF-36) questionnaires, decreases as CHB progresses to its more severe stages, from chronic inactive hepatitis to HCC. ${ }^{6}$ In addition, depression and anxiety were also more common in patients with CHB than in healthy controls.

However, the majority of previous research has involved cross-sectional studies, and there is a general lack of longitudinal data. Only one prospective study has been published that shows that subjects with CHB had an improvement in HRQoL after 24 weeks of antiviral treatment. ${ }^{8}$ This improvement was more obvious in females and hepatitis B e antigen ( $\mathrm{HBeAg}$ )-positive subjects. In addition, few studies have addressed the predictors or risk factors for low HRQoL and depression in patients with CHB.

Drug withdrawal is another key problem in CHB management. There are several problems associated with long-term NUC treatment, including development of drug resistance, poor adherence, economic burdens, and psychological stressors. ${ }^{910}$ The Asian Pacific Association for the Study of the Liver (APASL) Guidelines recommend that NUC treatment for $\mathrm{CHB}$ could be considered successful after serum HBV DNA is undetectable, HBeAg seroconversion has occurred, and alanine aminotransferase levels have normalized for at least 12 months. Treatment is then completed after another 12 months (HBeAg-positive patients) or 18 months (HBeAg-negative patients) of medication. ${ }^{11}$ However, relapse occurs in $\sim 50 \%$ of HBV patients after withdrawal of NUCs, even when the recommendations are followed. ${ }^{12,13}$ European association for the study of the liver (EASL) guidelines recommend that discontinuation of NUC treatment can be considered when hepatitis B surface antigen (HBsAg) seroconversion occurs. ${ }^{14}$ However, the possibility of HBsAg seroconversion during NUC therapy is limited and requires at least a decade of treatment. But long-term NUC treatment may lead to low HRQoL in patients with CHB. Whether benefits from the improved HRQoL that occurs after drug discontinuation outweigh the risks of viral relapse is an unanswered question. Thus, in summary, we speculate whether the ongoing NUC treatment increases HRQoL in patients with $\mathrm{CHB}$ and whether treatment interruption in patients with $\mathrm{CHB}$ will correlate with depressive symptoms, which is measured by Beck Depression Inventory (BDI).

Therefore, as we begin to focus on the relationship between $\mathrm{MH}$ and the evolution of disease outcome, how antiviral drug treatment and withdrawal influence the QoL of patients with CHB needs to be addressed. Our study examines whether, 1) ongoing NUC treatment enhances the HRQoL of patients with $\mathrm{CHB}$, and 2) drug withdrawal benefits their psychological well-being.

\section{Patients and methods}

\section{Patients}

Patients treated for CHB with NUCs in the First Affiliated Hospital of Xiamen University were examined. The patients were included in our study when it was convenient and they fulfilled the inclusion criteria. We defined the diagnosis of $\mathrm{CHB}$ as serum HBsAg-positive for at least 6 months combined with a repeated or continued increase in the serum alanine aminotransferase level. ${ }^{14}$ Patients were excluded if they had cirrhosis; HCC; metabolic liver disease; co-infections with hepatitis $\mathrm{C}$ virus, hepatitis $\mathrm{D}$ virus, or HIV; autoimmune hepatitis; were suffering from heavy alcohol abuse; were pregnant; or were previously treated with interferon. Patients with CHB were enrolled in the treatment group if they were being treated with NUCs for the first time and were enrolled in the discontinuation group if they had fulfilled the criteria by stopping medication treatment. Sociodemographic details, such as gender, age, ethnicity, education level, and income, were collected for all participating subjects. The definition was as follows: i) level of education - primary, less than 6 years of formal school education; secondary, 6-12 years of formal school education; and tertiary, university and postgraduate studies; and ii) income - low, $<1,999 \mathrm{RMB} /$ month; middle, 2,0003,999 RMB/month; and high, >4,000 RMB/month.

The demographic and baseline characteristics of the two groups are shown in Table 1.

\section{Follow-up}

Of the 112 patients who met the enrollment criteria, 54 patients in the treatment group and 48 patients in the discontinuation 
Table I Characteristics of patients in the treatment group and discontinuation group

\begin{tabular}{|c|c|c|}
\hline Characteristics & $\begin{array}{l}\text { Treatment } \\
\text { group }\end{array}$ & $\begin{array}{l}\text { Discontinuation } \\
\text { group }\end{array}$ \\
\hline \multicolumn{3}{|l|}{ Gender } \\
\hline Male & 40 (74.I) & $4 \mathrm{I}(85.4)$ \\
\hline Female & 14 (25.9) & $7(14.6)$ \\
\hline Age, years & $35.54 \pm 9.67$ & $37.77 \pm 7.48$ \\
\hline \multicolumn{3}{|l|}{ Marital status } \\
\hline Single & I 5 (27.8) & $7(14.6)$ \\
\hline Married & $39(72.2)$ & $4 I(85.4)$ \\
\hline \multicolumn{3}{|l|}{ Level of education } \\
\hline Primary & $4(7.4)$ & $5(10.4)$ \\
\hline Secondary & $40(74.1)$ & $28(58.3)$ \\
\hline Tertiary & $10(18.5)$ & $15(31.3)$ \\
\hline \multicolumn{3}{|l|}{ Income } \\
\hline Low & $6(12.5)$ & $3(5.6)$ \\
\hline Middle & $36(75.0)$ & $44(81.5)$ \\
\hline High & $6(12.5)$ & 7 (12.9) \\
\hline
\end{tabular}

Notes: Level of education: primary, $<6$ years of formal school education; secondary, 6-12 years of formal school education; and tertiary, university and postgraduate studies. Income: low, <I,999 RMB/month; middle, 2,000-3,999 RMB/month; and high, >4,000 RMB/month. Data presented as $n$ (\%) or mean \pm standard deviation.

group completed the 48-week follow-up. There were 10 patients in the discontination group who discontinued follow-up prematurely. Among them, three patients dropped out due to pregnancy and seven dropped out due to their request. The treatment group attended the Department of Infectious Diseases, First Affiliated Hospital of Xiamen University. Patients with CHB were asked to fill in the SF-36, the EQ-5D, and the BDI questionnaires. For the treatment group, the SF-36, EQ-5D, and BDI questionnaires were filled in at baseline and 48 weeks after starting the antiviral treatment. For the discontinuation group, the questionnaires were filled in when they discontinued treatment and again after 48 weeks.

All the subjects completed the questionnaire surveys in a quiet room without any interference or disruptions. Professional staff members were available to answer questions if any problems occurred with understanding the survey questions.

\section{Questionnaires}

\section{SF-36}

The SF-36 is a brief self-administered HRQoL instrument commonly used in various disease populations. It includes eight items: physical functioning, role limitations due to physical problems, bodily pain, general health, vitality, social functioning, role limitations due to emotional problems, and MH. In addition, there are two summary measures: the physical component summary and mental component summary scales. Items with higher scores mean better health conditions. The Chinese version of the SF-36 questionnaire is available and provided by the developer. The tool's validity and screening ability have been documented in various samples in the People's Republic of China. ${ }^{15,16}$

\section{EQ-5D}

The EQ-5D self-reported questionnaire has been used as an outcome measure in many international clinical trials. The Chinese (Taiwanese) version of the EQ-5D questionnaire was authorized by the EuroQol Group in 2000. Five dimensions were included: mobility, self-care, usual activities, pain/discomfort, and anxiety/depression. Each item has one question, with the following possible ratings: no problem, a moderate problem, or an extreme problem. In addition, a visual analog scale (VAS) was also included for the patients to summarize their overall health status, with higher scores indicating worse health. Thus, we found that the EQ-5D is a useful instrument for surveying a large population with relatively low education levels.

\section{$\mathrm{BDI}$}

The BDI is widely used for assessing depression severity with high reliability and efficacy. It is a self-reporting measure involving 21 items. Each item is rated on a scale ranging from 0 to 3, and the average provides a global depression score, with higher scores representing greater severity of depression.

We used two stable HRQoL questionnaires and a psychometric inventory in our study that complemented each other. EQ-5D was used to calculate quality-adjusted life years directly. Conversely, SF-36 provided a more comprehensive and multidimensional assessment of HRQoL. Hence, SF-36 and EQ-5D provided different information, which enabled the HRQoL evaluation of HBV patients to be more comprehensive and informative.

\section{Statistical analysis}

Continuous variables were expressed as mean and standard deviation, and categorical variables were expressed as percentages. The chi-square test and paired Student's $t$-test were applied as appropriate to determine statistically significant differences. Univariate (logistic regression) and multivariate analyses (stepwise multivariate logistic regression) were used to find factors associated with QoL. In addition, we constructed receiver-operating characteristic curves for determining the factors predicting QoL. The statistical significance of all tests was set as $P<0.05$ by two-tailed tests. Data analyses and quality control procedures were performed 
using SPSS for Windows, version 13.0 (SPSS Inc., Chicago, IL, USA).

\section{Ethical approval}

The Institutional Review Board of First Affiliated Hospital of Xiamen University approved this study. All procedures followed were in accordance with the ethical standards of the responsible committee on human experimentation and with the 1975 Declaration of Helsinki, as revised in 2008. Written informed consent was obtained from all patients before inclusion in the study.

\section{Results}

\section{Demographic data}

A total of 102 patients completed the SF-36 and EQ-5D questionnaires at follow-up visits; 54 subjects were in the treatment group and 48 subjects were in the drug discontinuation group. The characteristics of the subjects are shown in Table 1. All the patients were Chinese, and $80 \%$ of them were males.

\section{Dynamic change of HRQoL in treatment group and discontinuation group}

\section{SF-36 score}

Physical aspects of QoL, including physical functioning, physical role functioning, bodily pain, and general health perceptions, were significantly increased after the treatment, but the psychological measures, including vitality, social role functioning, emotional role functioning, and $\mathrm{MH}$, did not significantly increase after the antiviral drug treatment (Figure 1). There were no significant differences between the

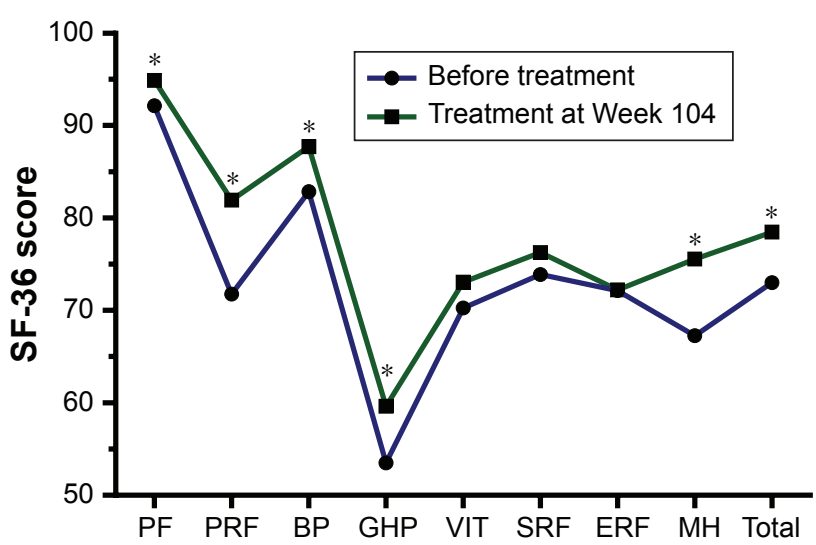

Figure I SF-36 scores for patients before and after antiviral treatment. Notes: The physical functions, including PF, PRF, BP, and GHP, were significantly increased after the treatment, while the psychological functions, including VIT, SRF, and ERF, had no obvious improvement after the antiviral treatment. $* P<0.05$. Abbreviations: SF-36, 36-Item Short-Form Health Survey; PF, physical functioning; PRF, physical role functioning; BP, bodily pain; GHP, general health perceptions; VIT, vitality; SRF, social role functioning; ERF, emotional role functioning; $\mathrm{MH}$, mental health. patients before discontinuing treatment and the patients ending treatment after 52 weeks in most of the SF-36 scales, with the exception of general health perception, emotional role functioning, and $\mathrm{MH}$, where the patients who discontinued treatment had significantly higher scores than the patients who continued NUCs during the study period (Figure 2).

\section{EQ-5D score}

For the patients treated with NUCs, high percentages reported improved mobility, ability to do normal activities, and pain relief after treatment, while the percentage of patients with depression did not decrease. In contrast, lower percentages of patients complained of depression and pain after completion of treatment, compared to before discontinuing treatment (Table 2).

\section{VAS score}

The VAS score, indicating subjective health status, was significantly increased after the NUC treatment, compared to the baseline values $(76.4 \pm 14.9$ vs $85.1 \pm 12.6, P=0.012)$. However, there was no significant improvement or decrease after patients discontinued treatment after 52 weeks $(83.7 \pm 11.9$ vs $83.7 \pm 13.4, P=0.36$; Figure 3 ).

\section{BDI}

When we compared the patients before and after the NUC treatment, no significant decrease in the BDI score occurred after treatment. However, the BDI score was significantly decreased after medication treatment was discontinued $(6.6 \pm 4.9$ vs $4.07 \pm 4.9, P=0.046)$, suggesting that the severity

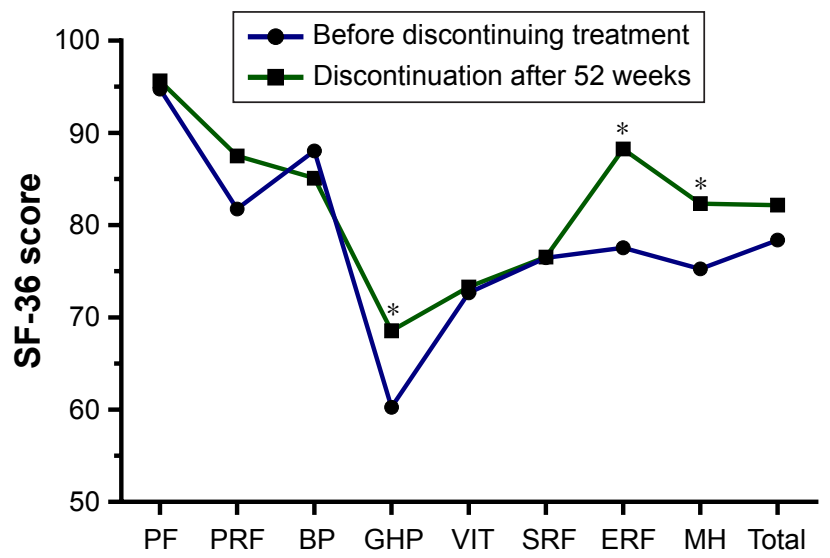

Figure 2 SF-36 scores for patients before and after discontinuation of treatment. Notes: There were no significant differences between patients before discontinuing treatment and patients who discontinued treatment after 52 weeks in almost all the SF-36 scales, with the exception of GHP, ERF, and MH scales, where patients who discontinued treatment had significantly higher scores than patients had before discontinuing treatment. $* P<0.05$.

Abbreviations: SF-36, 36-Item Short-Form Health Survey; GHP, general health perceptions; ERF, emotional role functioning; $\mathrm{MH}$, mental health; $\mathrm{PF}$, physical functioning; PRF, physical role functioning; BP, bodily pain; VIT, vitality; SRF, social role functioning. 
Table 2 EQ-5D scores for patients in each group

\begin{tabular}{|c|c|c|c|c|c|}
\hline \multirow[t]{3}{*}{ Dimension } & \multirow[t]{3}{*}{ Level } & \multicolumn{2}{|c|}{$\begin{array}{l}\text { Treatment } \\
\text { group }\end{array}$} & \multicolumn{2}{|c|}{$\begin{array}{l}\text { Discontinuation } \\
\text { group }\end{array}$} \\
\hline & & Before & After & Before & After \\
\hline & & n (\%) & n (\%) & n (\%) & n (\%) \\
\hline \multirow[t]{3}{*}{ Mobility } & 1 & $34(89.5)$ & $38(100)^{*}$ & $28(100)$ & $28(100)$ \\
\hline & 2 & $4(10.5)$ & 0 & 0 & 0 \\
\hline & 3 & 0 & 0 & 0 & 0 \\
\hline \multirow[t]{3}{*}{ Self-care } & 1 & $37(97.4)$ & $38(100)$ & $28(100)$ & $28(100)$ \\
\hline & 2 & $I(2.6)$ & 0 & 0 & 0 \\
\hline & 3 & 0 & 0 & 0 & 0 \\
\hline \multirow[t]{3}{*}{ Usual activities } & 1 & $32(84.2)$ & $38(100)^{*}$ & $28(100)$ & $28(100)$ \\
\hline & 2 & $6(15.8)$ & 0 & 0 & 0 \\
\hline & 3 & 0 & 0 & 0 & 0 \\
\hline \multirow[t]{3}{*}{ Pain/discomfort } & I & $25(65.8)$ & $34(89.5)^{*}$ & $21(75.0)$ & $27(96.4)^{*}$ \\
\hline & 2 & $12(31.6)$ & $4(10.5)$ & $7(25.0)$ & I (3.6) \\
\hline & 3 & $\mathrm{I}(2.6)$ & 0 & 0 & 0 \\
\hline \multirow[t]{3}{*}{ Anxiety/depression } & 1 & $28(73.7)$ & $29(76.3)$ & $20(7 \mid .4)$ & $27(96.4)^{*}$ \\
\hline & 2 & $10(26.3)$ & $9(23.7)$ & $8(28.6)$ & I (3.6) \\
\hline & 3 & 0 & 0 & 0 & 0 \\
\hline
\end{tabular}

Notes: Data are presented with number (percentage). Level I, no problem; level 2, some problem; and level 3, severe problem. $* p<0.05$.

Abbreviation: EQ-5D, European Quality of Life-5 Dimensions.

of depression is alleviated after the completion of pharmacotherapy (Figure 4).

\section{Factors associated with HRQoL after intervention}

To explore the relationships between baseline characteristics and the SF-36 scores after intervention, univariate analyses were performed and the results showed that the type of intervention $(P=0.007)$ and the baseline SF-36 score $(P<0.001)$ were the predictors for QoL. Multivariate logistic regression

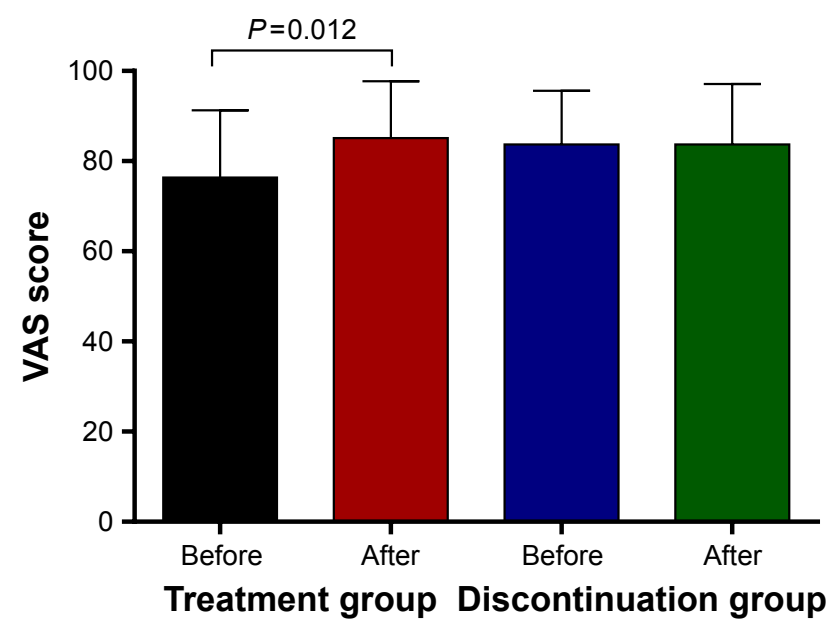

Figure 3 The VAS score for health condition of patients.

Notes: The VAS score, indicating health condition subjectively, was significantly increased after the antiviral treatment $(85.1 \pm 12.6)$, compared to patients before treatment $(76.4 \pm 14.9, P=0.012)$. However, there was no significant improvement or decrease after patients discontinued treatment after 52 weeks $(83.7 \pm 1$ I.9 vs 83.7 $\pm 13.4, P=0.36$ ).

Abbreviation: VAS, visual analog scale.

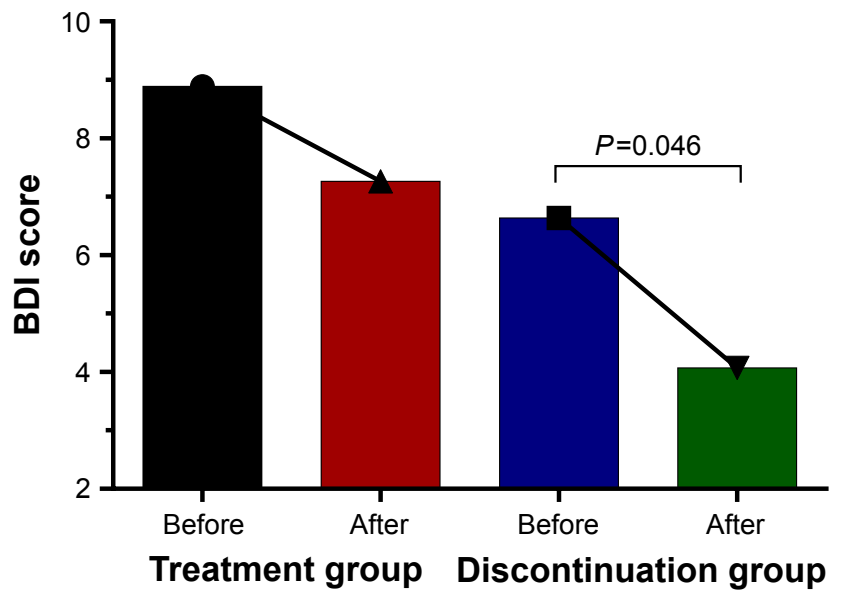

Figure 4 The BDI score for patients.

Notes: When the patients were compared before and after antiviral treatment, no significant decrease in the BDI score was observed ( $8.9 \pm 6.6$ vs $7.3 \pm 5.5)$. However, the $\mathrm{BDI}$ score significantly decreased after the treatment discontinuation $(6.6 \pm 4.9 \mathrm{vs}$ $4.07 \pm 4.9, P=0.046$ ), suggesting that discontinuing the treatment would alleviate the severity of depression.

Abbreviation: BDI, Beck Depression Inventory.

analysis of the SF-36 score included baseline characteristics and type of treatment (Table 3). Baseline SF-36 scores were strong predictors for improvement in HRQoL after intervention. Patients with CHB tended to achieve higher QoL after intervention if they had higher baseline SF-36 scores (odds ratio $=1.17,95 \%$ confidence interval $[\mathrm{CI}]$ : $1.06-1.29, P=0.003)$.

To validate the results, the receiver-operating characteristic curve was calculated. The area under the receiveroperating characteristic curve using the baseline SF-36 score to predict improvement in HRQoL was 0.84 (95\% CI: $0.74-0.93, P<0.001$; Figure 5). When the cutoff value for SF-36 was set at 79.5, the sensitivity and specificity for predicting QoL improvement were $82.8 \%$ and $74.0 \%$, respectively.

\section{Factors predicting the alleviation of depression}

We also explored the relationships between baseline characteristics and depression. The univariate analysis showed that the baseline VAS score $(P=0.03)$ and the baseline BDI score ( $P=0.001)$ predicted alleviation of depression. Multivariate logistic regression analysis showed that the baseline BDI score predicted risk of the patients suffering from depression (odds ratio $=0.75,95 \%$ CI: $0.62-0.92, P=0.005$ ), suggesting that patients were less likely to get remission of depression when they have a lower baseline BDI score (Table 4). The receiver-operating characteristic for baseline BDI predicts depression alleviation after the intervention $(0.78,95 \% \mathrm{CI}$ : $0.66-0.89, P<0.001)$. The optimal cutoff value was 8.5 , and 
Table 3 Factors associated with HRQoL after intervention

\begin{tabular}{|c|c|c|c|c|c|c|}
\hline \multirow[t]{2}{*}{ Variables } & \multicolumn{3}{|c|}{ Univariate analysis } & \multicolumn{3}{|c|}{ Multivariate analysis } \\
\hline & OR & $95 \% \mathrm{Cl}$ & $P$-value & OR & $95 \% \mathrm{Cl}$ & $P$-value \\
\hline Gender & 1.34 & $0.48-3.76$ & 0.58 & 0.83 & $0 .|2-5.8|$ & 0.85 \\
\hline Age, years & 1.03 & $0.98-1.08$ & 0.32 & 1.05 & $0.95-1.15$ & 0.35 \\
\hline Marital status & 3.05 & $0.83-11.24$ & 0.09 & 1.26 & $0.15-10.75$ & 0.83 \\
\hline Level of education & 0.67 & $0.31-1.47$ & 0.67 & 0.53 & $0.23-1.21$ & 0.13 \\
\hline Income & 0.48 & $0.18-1.28$ & 0.14 & 0.22 & $0.05-1.06$ & 0.06 \\
\hline Type of intervention ${ }^{\mathrm{a}}$ & 0.28 & $0.11-0.71$ & 0.007 & 0.66 & $0.13-3.33$ & 0.61 \\
\hline Total SF-36 score & 1.16 & $1.08-1.24$ & $<0.001$ & 1.17 & $1.06-1.29$ & 0.003 \\
\hline VAS score & 1.01 & $0.97-1.04$ & 0.79 & 0.96 & $0.90-1.03$ & 0.25 \\
\hline BDI score & 0.98 & $0.89-1.07$ & 0.59 & 0.94 & $0.81-1.09$ & 0.39 \\
\hline
\end{tabular}

Note: ${ }^{\mathrm{T} T y p e}$ of intervention indicated antiviral treatment or discontinued antiviral treatment.

Abbreviations: HRQoL, health-related quality of life; OR, odds ratio; Cl, confidence interval; SF-36, 36-Item Short-Form Health Survey; VAS, visual analog scale; BDI, Beck Depression Inventory.

the sensitivity and specificity values for predicting depression remission were $58.6 \%$ and $76.0 \%$, respectively. Patients with BDI score $<8.5$ at baseline had a higher chance of depression remission after the intervention (Figure 6).

\section{Discussion}

Patients with CHB suffered from impaired physical health often accompanied by depressive symptoms. ${ }^{17}$ HRQoL of patients with $\mathrm{CHB}$ was obviously inferior to that of the general population. ${ }^{18,19}$ NUCs have been widely used to treat $\mathrm{CHB}$ and prevent the development of end-stage liver disease, but their effects on HRQoL have not been fully documented. This study is the first to observe the change in SF-36, ED-5Q, and BDI after the initiation and termination of antiviral treatment in patients with $\mathrm{CHB}$. We demonstrated

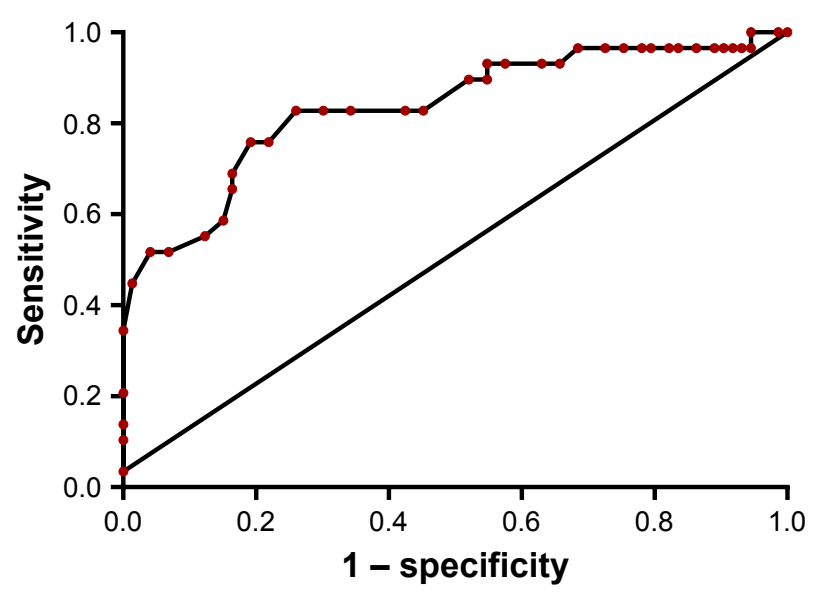

Figure 5 ROC curves of baseline SF-36 score in predicting the life quality. Notes: The ROC curve for SF-36 was 0.84 (95\% Cl: 0.74-0.93). When the cutoff value for SF-36 was set at 79.5, the sensitivity and specificity for predicting QoL were $82.8 \%$ and $74.0 \%$, respectively. The QoL improvement after medical intervention was associated with the baseline SF-36 score.

Abbreviations: ROC, receiver-operating characteristic; SF-36, 36-Item Short-Form Health Survey; $\mathrm{Cl}$, confidence interval; QoL, quality of life. that NUC pharmacotherapy benefits physical health but not psychological status, especially in those with high SF-36 scores and/or low BDI scores at baseline. Patients with previous history of $\mathrm{MH}$, heavy alcohol consumption, and drug abuse need more attention paid to their psychological status during NUC antiviral treatment since studies have reported that patients with mental disorder history, alcohol, and/or drug abuse have poor HRQoL and MH. ${ }^{20,21}$

Few studies have addressed the effects of NUC treatment on the HRQoL and $\mathrm{MH}$ in patients with CHB. It was suggested that stopping the antiviral drugs too early can lead to relapse. ${ }^{22}$ However, long-term treatment will also result in problems, including adverse drug effects, economic burdens, drug resistance, and psychological stressors. It is vital to balance the risks and benefits from continuing or terminating NUC pharmacotherapy. By analyzing outcomes in the patients who stopped antiviral medications according to guidelines from the APASL, our research showed that mental status improved significantly after cessation of NUC treatment, as shown by the EQ-5D anxiety score, the mental component of SF-36, and the BDI score. However, the multivariate logistic regression analysis indicated that the baseline SF-36 score is the only independent factor to predict which patients would obtain a better QoL irrespective of whether patients receive continued treatment or not. Our study demonstrated that patients with $\mathrm{CHB}$ have better QoL after interventions (continued treatment or not) if they had higher SF-36 scores at baseline. Given the risks of HBV relapse after termination of NUC treatment, continued antiviral therapy may be the more beneficial strategy for clinicians to advocate.

The present study also showed that mobility, pain, and the usual activities domain of the EQ-5D improved after 104 weeks of antiviral treatment. However, the anxiety 
Table 4 Factors associated with depression after intervention

\begin{tabular}{|c|c|c|c|c|c|c|}
\hline \multirow[t]{2}{*}{ Variables } & \multicolumn{3}{|c|}{ Univariate analysis } & \multicolumn{3}{|c|}{ Multivariate analysis } \\
\hline & OR & $95 \% \mathrm{Cl}$ & $P$-value & OR & $95 \% \mathrm{Cl}$ & $P$-value \\
\hline Gender & 0.49 & $0.12-1.99$ & 0.49 & 0.63 & $1.11-3.45$ & 0.59 \\
\hline Age, years & 0.99 & $0.94-1.04$ & 0.64 & 1.01 & $0.94-1.09$ & 0.77 \\
\hline Marital status & 0.94 & $0.31-2.87$ & 0.92 & 1.01 & $0.97-1.04$ & 0.93 \\
\hline Level of education & 0.82 & $0.37-1.77$ & 0.61 & 1.44 & $0.64-3.19$ & 0.38 \\
\hline Income & 0.62 & $0.22-1.76$ & 0.37 & 0.29 & $0.06-1.53$ & 0.15 \\
\hline Type of intervention ${ }^{a}$ & 0.90 & $0.33-2.46$ & 0.84 & 0.89 & $0.19-3.74$ & 0.83 \\
\hline Total SF-36 score & 0.97 & $0.93-1.01$ & 0.18 & 0.95 & $0.93-1.04$ & 0.61 \\
\hline VAS score & 1.05 & $1.01-1.09$ & 0.03 & 0.95 & $0.91-1.01$ & 0.09 \\
\hline BDI score & 0.77 & $0.66-0.91$ & 0.001 & 0.75 & $0.62-0.92$ & 0.005 \\
\hline
\end{tabular}

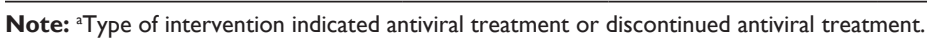

Abbreviations: OR, odds ratio; Cl, confidence interval; SF-36, 36-Item Short-Form Health Survey; VAS, visual analog scale; BDI, Beck Depression Inventory.

domain of EQ-5D was not increased, favoring the idea that patients with CHB may benefit from psychotherapy during antiviral treatment, which is often overlooked by the treating clinicians. This could be affected by the stage of disease progression, which was not determined in this survey, as well as the cultural and social context of the study populations.

Undoubtedly, several factors associated with HRQoL and depression in patients with CHB are not well understood. In addition to $\mathrm{HBV}$ effects on liver, patients with $\mathrm{CHB}$ may exhibit depression and reduction in their HRQoL. HBV infection could influence the central nervous system and cause cognitive dysfunction in many ways. ${ }^{23,24}$ However, the mechanism between behavioral disorders that negatively affect HRQoL and HBV chronic infection is still unknown. Previous studies have shown that disease severity, gender, $\mathrm{HBeAg}$

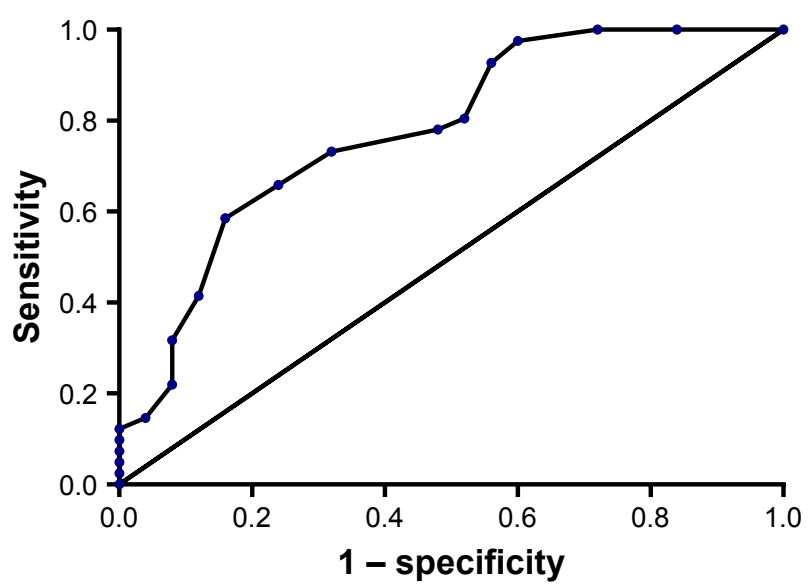

Figure 6 ROC curves of baseline BDI score in predicting the depression condition. Notes: The ROC curve for baseline BDI was 0.78 (95\% Cl: 0.66-0.89). The optimal cutoff value was found as 8.5 , and the sensitivity and specificity values for predicting depression condition were $58.6 \%$ and $76.0 \%$, respectively. Patients with higher BDI score at baseline had a higher risk of suffering from depression after medical intervention. Abbreviations: ROC, receiver-operating characteristic; BDI, Beck Depression Inventory; $\mathrm{Cl}$, confidence interval. positivity, alcohol consumption, age, and lack of awareness of CHB may be related to the HRQoL of patients during treatment. ${ }^{6,25,26}$ Kunkel et al ${ }^{27}$ reported an association between BDI scores, higher psychosocial stress, poorer general functioning, and higher liver enzyme levels. In multiple regression analyses, older age and lack of awareness were predictors of stigma scores with CHB. One pitfall in most studies was the failure to consider baseline HRQoL and depression severity. This study was the first to include the baseline SF-36 score and BDI score in the multiple regression analysis, and we found that the baseline SF-36 score and BDI score were important predictors of HRQoL and MH. Therefore, the baseline assessment of HRQoL and MH should be included in the routine evaluation of these patients.

Our study has clinical relevance as mental health problems were reported to be common among patients with chronic liver diseases and they can have significant health implications. ${ }^{18,28,29}$ Addressing depression and psychological problems with adequate interventions should improve the adjustment in patients with CHB. In addition, assessing $\mathrm{MH}$ at the initial encounter with the patient is useful, because individuals with low SF-36 scores and high BDI scores at baseline have a higher risk of having poor HRQoL and depression after treatment. Professional help, including educational or psychological interventions, should be given to these patients, as it has been shown that HRQoL of these patients significantly improves after a brief educational program. ${ }^{30}$

\section{Limitations}

There are some limitations of our study. First, we have not considered the severity of the liver disease, although different disease stages can affect HRQoL to different degrees. Second, in view of the small sample size of this study, caution should be exercised before generalizing the conclusions to all patients with CHB. 


\section{Conclusion}

Antiviral treatment with NUC benefits the physical health of the patients with $\mathrm{CHB}$, while conferring no obvious improvement in their psychological condition. Improved psychological interventions for patients with $\mathrm{CHB}$, especially for those with lower baseline SF-36 scores and higher BDI scores, may improve their QoL. Although discontinuing antiviral medications may improve their psychological status, the multivariate logistic regression analysis indicated that baseline SF-36 scores and BDI scores were the only independent factors that could predict whether the patients would obtain a better QoL and remission of depression, whether or not they received continued treatment. Considering the risk of HBV relapse after drug withdrawal, a continued antiviral treatment, with attendant psychological intervention, may be the more suitable clinical strategy.

\section{Acknowledgment}

We wish to thank Zhiyun Feng for his helpful assistance in the study.

\section{Disclosure}

The authors report no conflicts of interest in this work

\section{References}

1. Liaw YF, Chu CM. Hepatitis B virus infection. Lancet. 2009;373(9663): 582-592.

2. Sarin SK, Kumar M, Lau GK, et al. Asian-Pacific clinical practice guidelines on the management of hepatitis B: a 2015 update. Hepatol Int. 2016;10(1):1-98.

3. Cai S, Yu T, Jiang Y, Zhang Y, Lv F, Peng J. Comparison of entecavir monotherapy and de novo lamivudine and adefovir combination therapy in $\mathrm{HBe} A g$-positive chronic hepatitis B with high viral load: 48-week result. Clin Exp Med. 2016;16(3):429-436.

4. Terrault NA, Bzowej NH, Chang KM, Hwang JP, Jonas MM, Murad MH. AASLD guidelines for treatment of chronic hepatitis B. Hepatology. 2016;63(1):261-283.

5. Peng J, Yin J, Cai S, Yu T, Zhong C. Factors associated with adherence to nucleos(t)ide analogs in chronic hepatitis B patients: results from a 1-year follow-up study. Patient Prefer Adherence. 2015;9:41-45.

6. Ong SC, Mak B, Aung MO, Li SC, Lim SG. Health-related quality of life in chronic hepatitis B patients. Hepatology. 2008;47(4):1108-1117.

7. Altindag A, Cadirci D, Sirmatel F. Depression and health related quality of life in non-cirrhotic chronic hepatitis B patients and hepatitis B carriers. Neurosciences (Riyadh). 2009;14(1):56-59.

8. Kwon SY, Lee YS, Lee JH, Koo HW, Choi W, Lee CH, editors. Impact of Antiviral Treatment on Health-Related Quality of Life in Chronic Hepatitis B Patients: A Prospective Longitudinal Study 2008. Hoboken, NJ: John Wiley \& Sons Inc; 2008.

9. Peng J, Cao J, Yu T, et al. Predictors of sustained virologic response after discontinuation of nucleos(t)ide analog treatment for chronic hepatitis B. Saudi J Gastroenterol. 2015;21(4):245-253.

10. Cai SH, Lv FF, Zhang YH, Jiang YG, Peng J. Dynamic comparison between Daan real-time PCR and Cobas TaqMan for quantification of HBV DNA levels in patients with CHB. BMC Infect Dis. 2014;14:85.
11. Liaw YF, Kao JH, Piratvisuth T, et al. Asian-Pacific consensus statement on the management of chronic hepatitis B: a 2012 update. Hepatol Int. 2012;6(3):531-561.

12. Ahn SH, Chan HL, Chen PJ, et al. Chronic hepatitis B: whom to treat and for how long? Propositions, challenges, and future directions. Hepatol Int. 2010;4(1):386-395.

13. Fung J, Lai CL, Tanaka Y, et al. The duration of lamivudine therapy for chronic hepatitis B: cessation vs. continuation of treatment after $\mathrm{HBeAg}$ seroconversion. Am J Gastroenterol. 2009;104(8):1940-1946.

14. European Association for the Study of Liver. EASL clinical practical guidelines: management of alcoholic liver disease. J Hepatol. 2012; 57(2):399-420.

15. Chung J, Kwan A, Kwok J, Chan S. Health-related quality-of-life questionnaire for women with polycystic ovary syndrome: a Chinese translation and validation study. BJOG. 2016;123(10):1638-1645.

16. Zhang L, Tu L, Chen J, et al. Psychological distress as a crucial determinant for quality of life in patients with noncardiac chest pain in Central China: a cross-sectional study. Medicine (Baltimore). 2016;95(46):e5289.

17. Modabbernia A, Ashrafi M, Malekzadeh R, Poustchi H. A review of psychosocial issues in patients with chronic hepatitis B. Arch Iran Med. 2013;16(2):114-122.

18. Karaivazoglou K, Iconomou G, Triantos C, et al. Fatigue and depressive symptoms associated with chronic viral hepatitis patients. health-related quality of life (HRQOL). Ann Hepatol. 2010;9(4):419-427.

19. Bao ZJ, Qiu DK, Ma X, et al. Assessment of health-related quality of life in Chinese patients with minimal hepatic encephalopathy. World $J$ Gastroenterol. 2007;13(21):3003-3008.

20. Ndikuno C, Namutebi M, Kuteesa J, Mukunya D, Olwit C. Quality of life of caregivers of patients diagnosed with severe mental illness at the national referral hospitals in Uganda. BMC Psychiatry. 2016;16(1):400.

21. Lozano OM, Rojas AJ, Fernandez CF. Psychiatric comorbidity and severity of dependence on substance users: how it impacts on their health-related quality of life? J Ment Health. Epub 2016 Apr 29.

22. Wu X, Cai S, Li Z, et al. Potential effects of telbivudine and entecavir on renal function: a systematic review and meta-analysis. Virol $J$. 2016;13(1):64.

23. Lv XF, Qiu YW, Tian JZ, et al. Abnormal regional homogeneity of resting-state brain activity in patients with $\mathrm{HBV}$-related cirrhosis without overt hepatic encephalopathy. Liver Int. 2013;33(3):375-383.

24. Lv XF, Ye M, Han LJ, et al. Abnormal baseline brain activity in patients with HBV-related cirrhosis without overt hepatic encephalopathy revealed by resting-state functional MRI. Metab Brain Dis. 2013;28(3):485-492.

25. Spackman DE, Veenstra DL. A cost-effectiveness analysis of currently approved treatments for $\mathrm{HBeAg-positive} \mathrm{chronic} \mathrm{hepatitis} \mathrm{B.}$ Pharmacoeconomics. 2008;26(11):937-949.

26. Weinstein AA, Kallman PJ, Stepanova M, et al. Depression in patients with nonalcoholic fatty liver disease and chronic viral hepatitis $\mathrm{B}$ and $\mathrm{C}$. Psychosomatics. 2011;52(2):127-132.

27. Kunkel EJ, Kim JS, Hann HW, et al. Depression in Korean immigrants with hepatitis B and related liver diseases. Psychosomatics. 2000;41(6):472-480.

28. Foster GR, Goldin RD, Thomas HC. Chronic hepatitis C virus infection causes a significant reduction in quality of life in the absence of cirrhosis. Hepatology. 1998;27(1):209-212.

29. Modabbernia A, Ashrafi M, Keyvani H, et al. Brain-derived neurotrophic factor predicts physical health in untreated patients with hepatitis C. Biol Psychiatry. 2011;70(5):e31-e32.

30. SharifF, Mohebbi S, Tabatabaee HR, Saberi-Firoozi M, Gholamzadeh S. Effects of psycho-educational intervention on health-related quality of life (QOL) of patients with chronic liver disease referring to Shiraz University of Medical Sciences. Health Qual Life Outcomes. 2005;3:81. 
Patient Preference and Adherence

Dovepress

\section{Publish your work in this journal}

Patient Preference and Adherence is an international, peer-reviewed, open access journal that focuses on the growing importance of patient preference and adherence throughout the therapeutic continuum. Patient satisfaction, acceptability, quality of life, compliance, persistence and their role in developing new therapeutic modalities and compounds to optimize

Submit your manuscript here: http://www.dovepress.com/patient-preference-and-adherence-journa clinical outcomes for existing disease states are major areas of interest for the journal. This journal has been accepted for indexing on PubMed Central. The manuscript management system is completely online and includes a very quick and fair peer-review system, which is all easy to use. Visit http://www. dovepress.com/testimonials.php to read real quotes from published authors. 